\title{
Expanding maps on the circle and geodesic laminations
}

\author{
Víctor F. Sirvent
}

\begin{abstract}
In this article we consider a family of orientation preserving expanding maps on the circle. We associate to each member of this family a geodesic lamination on the disc, endowed with a transversal measure. The map on the circle induces an expanding dynamical systems on the lamination. We explore relations between the geometry of the lamination and the symbolic dynamics of the circle map. We present a list of open problems.
\end{abstract}

\section{Introduction}

Expanding maps on the circle and on the interval has been studied extensively in dynamical systems, since they exhibit important dynamical and ergodic properties (cf. [11]). On the other hand geodesic laminations on the disc have been used in different areas of mathematics: complex dynamics [10, 16], surface automorphism dynamics [6], hyperbolic geometry [5] and symbolic dynamics [12, 13]. In $[4,9,10,16]$, and references within, geodesic laminations on the disc were associated to the angle doubling map on the circle as model of Julia sets.

In the present article we consider a family of expanding maps on the circle, these maps are orientation preserving and of degree $k$, with $k \geq 3$. This family of maps is defined in Section 2. In Section 3, we associate a geodesic lamination on the disc, for each map of the family. We define a transversal measure. The expanding map on the circle induces an expanding dynamical system on the lamination, see Theorem 3.1. Moreover, we show relations between the geometry of

Received by the editors in January 2014.

Communicated by P. De Maesschalck.

2010 Mathematics Subject Classification : 37E10, 37B99, 53C22, 28A80.

Key words and phrases : Expanding dynamical systems, geodesic laminations. 
the lamination and the dynamics of the expanding map on the circle, in particular to its symbolic dynamics.

In $[12,14,15]$ the author considered a family of fractals that can be described in term of space-filling curves, which can be modelled using geodesic laminations on the disc. These constructions are related to expanding maps on the circle. These maps belong to the family of expanding maps considered in the present article. Unlike those maps, in the present article we do not require the existence of a space-filling curve. Therefore the results of the present paper are in a more general setting than the one considered in $[12,14]$. Some of the techniques used in the present paper are based in techniques considered by the author in $[12,14]$.

In Section 4, we consider some examples. We end the present article with Section 5, where we present a list of open problems.

\section{Expanding maps on the circle}

Let $I=[0,1)$ be the unit interval, we define a map $f: I \rightarrow I$ in the following way:

1. Let $\left\{I_{1}, \ldots, I_{k}\right\}$ be a partition of $I$, where $I_{j}$ are half-open intervals, closed to the left.

2. For $1 \leq j \leq k$, let $\phi_{j}: I_{j} \rightarrow I$ be $C^{1}$ surjective maps on the closure of $I_{j}$ with $\phi_{j}^{\prime}(t)>1$, for all $t \in \overline{I_{j}}$.

3. For a fixed $\theta \in(0,1)$, we define $R_{\theta}: I \rightarrow I$ as $R_{\theta}(t):=t+\theta(\bmod 1)$.

4. Let $f(t):=R_{\theta} \circ \phi_{j}(t)$, if $t \in I_{j}$.

We call this class of maps $f: I \rightarrow I$, regular expanding map on $I$. A special and important case of these maps is when all the $\phi_{j}$ are linear maps, in this case, we say that $f$ is a regular expanding linear map on $I$. These maps were introduced in [14].

We remark, that if we identify $S^{1}$ with $I=[0,1)(\bmod 1)$, then $f$ defines a continuous orientation preserving map of degree $k$ on $\mathbb{S}^{1}$.

The inverse branches of the map $f$ are $h_{j}: I \rightarrow I_{j}$, defined as $h_{j}:=\phi_{j}^{-1} \circ R_{\theta}^{-1}=$ $\phi_{j}^{-1} \circ R_{1-\theta}$, for $1 \leq j \leq k$. The collection of maps $\left\{h_{1}, \ldots, h_{k}\right\}$ is called the system of branches of the inverse function of $f$. Let $v: I \rightarrow\{1, \ldots, k\}$, where $v(t)=i$ if $t \in I_{i}$. The itinerary of $t$ is the infinite sequence $a_{1} a_{2} \ldots$, where $a_{j}=v\left(f^{j-1}(t)\right)$, for all $j \geq 1$. By definition it has the property $t \in \cap_{n=1}^{\infty} h_{a_{1}} \circ \cdots \circ h_{a_{n}}(I)$. Let us remark that the itinerary of a point is well-defined since the intervals $I_{i}$ are half-open. However there might be more than one point with the same itinerary, since the maps $h_{j}$ are not continuous as a map from $I$ to $I_{j}$. The lack of continuity of the maps $h_{i}$-s plays an important role in the construction of the lamination described in Section 3.

We say that a point $t$ is periodic or $f$-periodic if there exists a positive integer $m$, such that $f^{m}(t)=t$. In this case the itinerary of $t$ is of the form: $a_{1} \ldots a_{m} a_{1} \ldots a_{m}$ $a_{1} \ldots a_{m} \ldots$. We denote this itinerary by $\overline{a_{1} \cdots a_{m}}$. We say that $t$ is pre-periodic or $f$-pre-periodic, if $t$ is not periodic and there exists a positive integer $l$ such that 
$f^{l}(t)$ is periodic. In this case the itinerary of $t$ is of the form $a_{1} \ldots a_{l} \overline{a_{l+1} \cdots a_{l+m}}$, for some positive integer $m$.

Let the intervals $I_{j}$ be of the form $\left[t_{j}, t_{j+1}\right)$, and $\alpha_{j}=t_{j+1}-t_{j}$, i.e. its Lebesgue measure. By the definition of the map $f, h_{j}(\theta)=t_{j}$, i.e. $f\left(t_{j}\right)=\theta$, for $1 \leq j \leq k$.

Throughout the article we shall denote $I_{a_{1} \ldots a_{n}}:=h_{a_{1}} \circ \cdots \circ h_{a_{n}}(I)$ and $h_{a_{1} \ldots a_{n}}:=$ $h_{a_{1}} \circ \cdots \circ h_{a_{n}}$. We call the sets $I_{a_{1} \ldots a_{n}}$ cylinders of $I$. These sets consist of a finite union of half-open intervals, by the definition of the maps $h_{i}$. The total Lebesgue measure of $I_{a_{1} \ldots a_{n}}$ goes to zero as $n$ growths, in particular, if the the maps $\phi_{j}$-s are linear the Lebesgue measure of $I_{a_{1} \ldots a_{n}}$ is $\alpha_{a_{1}} \cdots \alpha_{a_{n}}$. We denote the collection of all cylinders by $\mathcal{I}$. i.e.

$$
\mathcal{I}:=\left\{I_{a_{1} \ldots a_{n}}: n \geq 1,1 \leq a_{i} \leq k\right\} .
$$

The extreme points or extremities of a cylinder are the boundary points of the intervals that form the cylinder. We call neighbouring extreme points of a cylinder $J$, a pair $x_{1}, x_{2}$ of extreme points of $J$ such that $x_{1} \in J$ and $x_{2} \in \bar{J} \backslash J$; and one the of the circle intervals, considered with the standard orientation, $\left(x_{2}, x_{1}\right),\left(x_{1}, x_{2}\right)$ is disjoint of $J$. For example, if the cylinder $J$ is of the form

$$
J=\left[y_{1}, y_{2}\right) \cup\left[y_{3}, y_{4}\right) \cup\left[y_{5}, y_{6}\right) ;
$$

the pairs of extreme points are $y_{1}, y_{6} ; y_{3}, y_{2}$ and $y_{5}, y_{4}$. Neighbouring extreme points come from the discontinuity point, of the maps $h_{i}{ }^{\prime}$ s, i.e. the point $\theta$.

Proposition 2.1. Let $I_{a_{1} \ldots a_{n}}$ be a cylinder and the pair $x_{1}, x_{2}$ be neighbouring extreme points of the cylinder, such that $x_{1} \in I_{a_{1} \ldots a_{n}}$ and $x_{2} \in \overline{I_{a_{1} \ldots a_{n}}} \backslash I_{a_{1} \ldots a_{n}}$. Then $x_{1}=t_{i}$ and $x_{2}=t_{i+1}$ or $x_{1}=h_{a_{1} \ldots a_{n^{\prime}}}\left(t_{i}\right)$ and $x_{2}=\lim _{t \rightarrow t_{i}^{-}} h_{a_{1} \ldots a_{n^{\prime}}}(t)=h_{a_{1} \ldots a_{n^{\prime}}}\left(t_{i+1}\right)$, for some $i \in\{1, \ldots, k\}, 1 \leq n^{\prime}<n$, here $i+1$ is taken mod $k$.

Proof. We use induction on $n$. If $n=1$, then the cylinder is $I_{a_{1}}=\left[t_{a_{1}}, t_{a_{1}+1}\right)$, so its neighbouring extreme points are $t_{a_{1}}$ and $t_{a_{1}+1}$. We suppose that the statement is true for $n=m$. Let $n=m+1$. The cylinder $I_{a_{1} \ldots a_{m}}$ is decomposed into subcylinders as follows:

$$
I_{a_{1} \ldots a_{m}}=\bigcup_{i=i}^{k} I_{a_{1} \ldots a_{m} i} .
$$

We shall check that the statement is true for $I_{a_{1} \ldots a_{m} i}$. Since $I_{a_{1} \ldots a_{m} i}=h_{a_{1} \ldots a_{m} i}(I)$, the points $h_{a_{1} \ldots a_{m}}\left(t_{i}\right), \lim _{t \rightarrow t_{i}^{-}} h_{a_{1} \ldots a_{m}}(t)$ are neighbouring extreme points of the cylinder $I_{a_{1} \ldots a_{m} i}$. If this cylinder has more than one connected component, the other neighbouring extreme points are neighbouring extreme points of the parent cylinder, i.e. $I_{a_{1} \ldots a_{m}}$, since the only discontinuity point of the map $h_{a_{1} \ldots a_{m} i}$ is $\theta$. Therefore the statement is true for $I_{a_{1} \ldots a_{m} i}$.

\section{Geodesic laminations}

Let $\mathbb{D}^{2}$ be the closed unit disk in the plane, and $S^{1}$ its boundary. We identify $S^{1}$ with $I=[0,1)$, since the map $f$ is well defined on $S^{1}$. We think this map as acting on the boundary of the disk.

A geodesic in $\mathbb{D}^{2}$ is an arc of circle that meets the boundary of $\mathbb{D}^{2}$ perpendicularly. 
Definition 3.1. A geodesic lamination on $\mathbb{D}^{2}$ is a non-empty closed set of geodesics of the disk and that any two of these geodesics do not intersect except at their end points.

The construction of the geodesic lamination $\Lambda$ is as follows: We consider the extremities of the intervals $I_{i}{ }^{\prime}$ s. And we join pairwise consecutive extremities by geodesics in $\mathbb{D}^{2}$. Let $a_{1} \ldots a_{n}$ be a word in the alphabet $\{1, \ldots, k\}$. We join by geodesics the points $h_{a_{1}} \cdots h_{a_{n}}\left(t_{j}\right)$ and $h_{a_{1}} \cdots h_{a_{n}}\left(t_{j+1}\right)$ for $j=0, \ldots, k$, where $j+1$ is taken $\bmod k$. We do this for all possible finite words in this alphabet and later we take the closure in the topology given by the Hausdorff metric of $\mathbb{D}^{2}$. We denote the set obtained in this way by $\Lambda$. The elements obtained in this way are either geodesics of $\mathbb{D}^{2}$ or points of $\mathbb{S}^{1}$; in the latter case the points are called degenerate geodesics. Due to Proposition 2.1, the set $\Lambda$ is formed by the closure in the Hausdorff metric of $\mathbb{D}^{2}$ of the set of geodesics that join neighbouring extreme points of all elements of $\mathcal{I}$. Figure 1 shows the set $\Lambda$, corresponding to Example 1 of Section 4. The set $\Lambda$ form a geodesic lamination, the proof is the same as in the case of a regular expanding linear map on $I$, done in [14]. For completeness sake, we present it here.



Figure 1: Geodesic lamination of Example 1, with $\alpha=(\sqrt{5}-1) / 2$ and $\beta=\alpha^{2}$.

Proposition 3.1. The set $\Lambda$ is a geodesic lamination on $\mathbb{D}^{2}$.

Proof. Let $J=I_{a_{1} \cdots a_{n}}$ be a cylinder and suppose that it consists of more than one connected component. Let $x_{1}$ and $x_{2}$ be neighbouring end points of this cylinder belonging to different connected components, see Figure 2. Due to the proof of Proposition 2.1 we can suppose, without loss of generality, that $x_{2}=h_{a_{1} \cdots a_{n}}(\theta)$ and $x_{1}=h_{a_{1} \cdots a_{n}}\left(\theta^{-}\right):=\lim _{t \rightarrow t^{*-}} h_{a_{1} \cdots a_{n}}(t)$. So $x_{1}=h_{a_{1} \cdots a_{n-1}}\left(t_{a_{n}+1}\right)$ and $x_{2}=$ $h_{a_{1} \cdots a_{n-1}}\left(t_{a_{n}}\right)$ where the sum $a_{n}+1$ is taken $\bmod k$.

Let us consider the cylinder $J^{\prime}=I_{a_{1} \cdots a_{n-1} a_{n}+1}$ whose left end point is $x_{1}$. We shall show that $J^{\prime}$ is contained in the gap of $J$ formed by $\left[x_{1}, x_{2}\right)$. Let us suppose 
that the cylinder $J^{\prime}$ consists of more than one connected component and one of these components is outside the gap $\left[x_{1}, x_{2}\right)$, say to the right of $x_{2}$. So there exists $1 \leq j \leq k$ such that $h_{a_{1} \cdots a_{n-1} a_{n}+1}\left(t_{j}\right)$ is the discontinuity point for this cylinder, i.e.

$$
x_{1}<y_{1}=h_{a_{1} \cdots a_{n-1} a_{n}+1}\left(t_{j}^{-}\right)<x_{2}<y_{2}=h_{a_{1} \cdots a_{n-1} a_{n}+1}\left(t_{j}\right) \text {. }
$$

So

$$
\begin{aligned}
h_{a_{1} \cdots a_{n-1}}\left(t_{a_{n}+1}\right)<h_{a_{1} \cdots a_{n-1} a_{n}+1}\left(t_{j+1}\right)<h_{a_{1} \cdots a_{n-1}}\left(t_{a_{n}}\right)<h_{a_{1} \cdots a_{n-1} a_{n}+1}\left(t_{j}\right) \\
h_{a_{1} \cdots a_{n-1} a_{n}+1}(\theta)<h_{a_{1} \cdots a_{n-1} a_{n}+1}\left(t_{j+1}\right)<h_{a_{1} \cdots a_{n-1} a_{n}}(\theta)<h_{a_{1} \cdots a_{n-1} a_{n}+1}\left(t_{j}\right) .
\end{aligned}
$$

Since the maps $h_{i}$ preserve the cyclic order of the images of $\left\{t_{1}, \ldots, t_{k}\right\}$. We have that the points $h_{a_{n}+1}(\theta), h_{a_{n}+1}\left(t_{j+1}\right), h_{a_{n}}(\theta)$ and $h_{a_{n}+1}\left(t_{j}\right)$ are in this cyclic order in $S^{1}$. However the first two points and the last belong to the interval $I_{a_{n}+1}$ and the third point to $I_{a_{n}}$ that contradicts the fact that these are disjoint intervals.



Figure 2: Figure relative to the proof of Proposition 3.1.

Proposition 3.2. Every point in I is an end point of a geodesic in $\Lambda$.

Proof. If the point $t$ belongs to the backward $f$-orbit of $\theta$, i.e. $f^{m}(t)=\theta$ for some positive integer $m$. Then point $t$ is an extreme point of a cylinder. So by definition there is a geodesic in the lamination having $t$ as an end point.

If $t$ does not belong to the backward $f$-orbit of $\theta$. Let $a_{1} a_{2} \ldots$ be its itinerary, i.e. $f^{i-1}(t) \in I_{a_{i}}$, for $i \geq 1$. Let

$$
\mathcal{L}:=\bigcup_{n \geq 1}\left\{\lambda \in \Lambda: \lambda \text { geodesic that joins neighbouring end points of } I_{a_{1} \ldots a_{n}}\right\} .
$$

By the definition of the geodesic lamination $\Lambda$, there exists a sequence of elements of the set $\mathcal{L}:\left\{\lambda_{l}\right\}_{l \geq 1}$ such that $t$ is in the closure of the end points of $\lambda_{l}$. Since $\Lambda$ is closed then the closure of $\mathcal{L}$ is contained in $\Lambda$. Hence $t$ is an end point of an element of the closure of $\mathcal{L}$.

Proposition 3.3. Let $a_{1} a_{2} \ldots$ be the itinerary of $\theta$. If

(a) $a_{m} \neq a_{1}$, for all $m>1$, or

(b) $\theta$ is preperiodic and its itinerary is of the form: $a_{1} \ldots a_{l-1} \overline{a_{l} \ldots a_{m+l}}$ for some $m$ and $l$ with $m>l>0$ and $a_{1} \ldots a_{l} \neq a_{j} \ldots a_{j+l-1}$, for $l \leq j \leq l+m$.

Then $\theta$ is a degenerate geodesic of the lamination $\Lambda$. 
Proof. The conditions (a) and (b) on the itinerary $\theta$ imply that $\theta$ is not $f$-periodic in either case.

First, we shall prove that the point $\theta$ is in the interior of the cylinders $I_{a_{1} \cdots a_{n}}$, for all $n>0$. If $\theta$ is not in the interior of $I_{a_{1} \cdots a_{n}}$, for some $n$; then by Proposition 2.1, $\theta=h_{a_{1} \ldots a_{n^{\prime}}}\left(t_{i}\right)$, for some $i \in\{1, \ldots, k\}$, and $n^{\prime}<n$. Hence $f^{n^{\prime}}(\theta)=$ $f^{n^{\prime}}\left(h_{a_{1} \ldots a_{n^{\prime}}}\left(t_{i}\right)\right)$, so $t_{i}=f^{n^{\prime}}(\theta)$. Therefore $\theta=f^{n^{\prime}+1}(\theta)$, contradicting the fact that $\theta$ is not periodic.

In the case (a), we shall prove, the cylinders of the form $I_{a_{1} \ldots a_{n}}$ consist of only one connected component for all $n$. We use induction, clearly $I_{a_{1}}$ has only one connected component. We suppose that $I_{a_{1} \ldots a_{n} a_{n+1}}$ has more than one connected component, while $I_{a_{1} \ldots a_{n}}$ consists of one connected component. Since $I_{a_{1} \ldots a_{n} a_{n+1}}$ has more than one connected component, the discontinuity point of $h_{a_{1}}$ is in the interior of $I_{a_{2} \ldots a_{n+1}}$ or $I_{a_{2} \ldots a_{n+1}}$ has more than one connected component. By definition of the maps $h_{i}, \theta$ is the discontinuity point for $1 \leq i \leq k$. Therefore in the former case $\theta \in I_{a_{2} \ldots a_{n+1}}$, so $a_{2}=a_{1}$, contradicting the hypothesis on the itinerary of $\theta$. In the later case, when $I_{a_{2} \ldots a_{n+1}}$ consists of more than one connected component. We repeat the previous argument, so we conclude that $\theta \in I_{a_{j} \ldots a_{n+1}}$, for some $j$, with $2 \leq j \leq n+1$. Hence $a_{j}=a_{1}$, contradicting the hypothesis on the itinerary of $\theta$. Therefore $I_{a_{1} \ldots a_{n}}$ consists of only one connected component for all $n$, when the hypothesis (a) is satisfied.

Let $\lambda_{n}$ the geodesic in $\Lambda$ that joins the extreme points of $I_{a_{1} \ldots a_{n}}$. By the first remark in this proof, $\theta$ is different of the ends points of $\lambda_{n}$, for all $n$. Since $I_{a_{1} \ldots a_{n}}$ consists of nested intervals whose length goes to zero, $\cap_{n \geq 1} I_{a_{1} \ldots a_{n}}$ consists of only one point, which is $\theta$. Therefore $\lambda_{n}$ converges to $\theta$ in the Hausdorff metric. Hence $\theta$ is an element of the lamination $\Lambda$.

In (b), by hypothesis $\theta \notin I_{a_{j} \ldots a_{j+l-1}}$, for $l \leq j \leq l+m$, so $\theta \notin I_{a_{j} \ldots a_{l+m}}$, then the cylinder $I_{a_{1} \ldots a_{l+m}}$ consists of only one connected component. Similarly for $I_{\left(a_{l} \ldots a_{l+m}\right)^{r}}$, for any positive $r$, where

$$
\left(a_{l} \ldots a_{l+m}\right)^{r}=\underbrace{a_{l} \ldots a_{l+m} \ldots a_{l} \ldots a_{l+m}}_{r \text { times }} .
$$

Since $\theta$ is the only discontinuity point of the maps $h_{i}-\mathrm{S}$, then $h_{a_{1} \ldots a_{l-1}}\left(I_{\left(a_{l} \ldots a_{l+m}\right)^{r}}\right)$, consists of only one connected component for all $r$.

By a similar argument used in the case (a), the geodesics that joins the end points of the interval $I_{a_{1} \ldots a_{l-1}\left(a_{l} \ldots a_{l+m}\right)^{r}}$, converges to $\theta$ in the Hausdorff metric, when $r$ goes to infinity. Hence $\theta$ is an element of the lamination $\Lambda$.

We introduce a dynamical system $(\Lambda, F)$, when $\theta$ is a degenerate geodesic (in particular when the hypotheses of Proposition 3.3 are satisfied), as follows: Let $\lambda$ be an element in $\Lambda$ whose end points are $t$ and $t^{\prime}$, we define $F(\lambda)$ as the geodesic that joins $f(t)$ and $f\left(t^{\prime}\right)$. The map $F$ is continuous since $f$ is continuous. If $\lambda$ joins $h_{a_{1}} \cdots h_{a_{n}}\left(t_{j}\right)$ with $h_{a_{1}} \cdots h_{a_{n}}\left(t_{j+1}\right)$ for $n \geq 1$ then $F(\lambda)$ joins $h_{a_{2}} \cdots h_{a_{n}}\left(t_{j}\right)$ with $h_{a_{2}} \cdots h_{a_{n}}\left(t_{j+1}\right)$. If $\lambda$ joins $t_{j}$ with $t_{j+1}$, let us consider $f\left(t_{j}\right)=R_{\theta} \phi_{j}\left(t_{j}\right)=R_{\theta}(0)=$ $\theta$ and similarly $f\left(t_{j+1}\right)=\theta$, and according to Proposition 3.3, the point $\theta$ is an element of $\Lambda$. Hence $F(\Lambda) \subset \Lambda$.

Let $\delta$ be any arc in $\mathbb{D}^{2}$ joining two distinct geodesics of the lamination, such that the intersection of these two geodesics with $\delta$ consists only of the end points 
of $\delta$ and the arc $\delta$ intersects each element of $\Lambda$ at most once. We call $\delta$ a transversal arc to $\Lambda$. We slide the arc $\delta$ along the geodesics towards the boundary of the disk according the two possible directions in which the geodesics can be oriented. With this procedure we obtain a limit set in the boundary of the disk, $C_{\delta}$. More precisely: Let $\lambda_{1}$ and $\lambda_{2}$ be the geodesics in $\Lambda$ that are joined by an arc $\delta$. This defines two disjoint intervals on the circle $J=\left[b_{1}, b_{2}\right]$ and $J^{\prime}=\left[b_{1}^{\prime}, b_{2}^{\prime}\right]$ where $b_{k}, b_{k}^{\prime}$ are the end points of $\lambda_{k}$ for $k=1,2$. Let $\lambda$ be a geodesic in the lamination such that its end points lie on the same interval $J$ or $J^{\prime}$, we remove from $J \cup J^{\prime}$ the open interval whose extremities are the end points of $\lambda$. The set $C_{\delta}$ is obtained in this way when all the geodesics in $\Lambda$ with end points in $J$ or $J^{\prime}$ are considered. See Figure 3. If $\delta$ is an arc, whose end points are geodesics that join extreme points of the same cylinder, then the set $C_{\delta}$ is obtained as $\cap_{j \geq 0} K_{j}$, where $K_{0}$ is the closure of the original cylinder and each $K_{j}$ is a union of closed sub-cylinders of $K_{0}$. By construction the set $C_{\delta}$ is not empty.

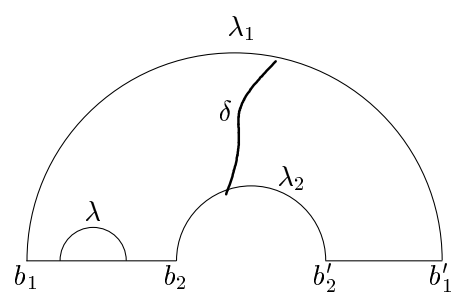

Figure 3: The construction of $C_{\delta}$.

Let $K_{0}=\overline{I_{a_{1} \ldots a_{m}}}$. Since $I_{a_{1} \ldots a_{m}}=\cup_{l=1}^{k} I_{a_{1} \ldots a_{m}}$, we have

$$
K_{1}=\overline{I_{a_{1} \ldots a_{m} b_{1}}} \cup \cdots \cup \overline{I_{a_{1} \ldots a_{m} b_{s}}}
$$

for some $s<k$ and $1 \leq b_{i} \leq k$. We remark that all the cylinders $I_{a_{1} \ldots a_{m} j}$ consisting of only one connected component are not in $K_{1}$. When we consider $K_{2}$, we have to see how each of these cylinders $I_{a_{1} \ldots a_{m} b_{i}}$ are subdivided into cylinders of the form $I_{a_{1} \ldots a_{m} b_{i} b_{i_{2}}}$. So $K_{2}=\cup_{i=1}^{S} \cup_{i_{2}=1}^{s_{i}} \overline{I_{a_{1} \ldots a_{m} b_{i} b_{i_{2}}}}$. Some of these cylinders could be sub-divided in the same way as the original cylinder $I_{a_{1} \ldots a_{m}}$, so we say that they are of the same type. In general, we say that two cylinders are of the same type if they are subdivided in a similar manner under this process. If the number of connected components of the family of cylinders $\mathcal{I}$ is bounded, there is a finite number of different types of cylinders. Hence, the set $C_{\delta}=\cap_{m \geq 1} K_{m}$, is the fixed point of a graph-directed iterated function system (GIFS). For definition and general properties of GIFS see [8, pp. 47]. If the map $f$ is a regular expanding linear map, then the contracting maps defined by the sub-division process of the cylinders are similarities, so the maps that define the GIFS are similarities. In Section 4 , we show in detail the construction of the limit set $C_{\delta}$ for some examples, see also $[12,14]$. We have proved the following proposition.

Proposition 3.4. If the number of the connected components of the family of cylinders $\mathcal{I}$ is bounded. Then the set $C_{\delta}$ is the fixed point of graph directed iterated function system (GIFS). Moreover the maps of this GIFS are similarities when $f$ is a regular expanding linear map. 
Proposition 3.5. If for some transversal arc $\delta$, the set $C_{\delta}$ is finite. Then the point $\theta$ is a f-periodic point.

Proof. Let $s_{1}, \ldots, s_{m}$ be the elements of $C_{\delta}$ and ordered such that $s_{i}<s_{i+1}$, for $1 \leq i \leq m-1$. So by definition $C_{\delta}=J \backslash \cup_{i=1}^{m-1} J_{i}$, where $J$ is the closure of an element of $\mathcal{I}$ and $J_{i}=\left(s_{i}, s_{i+1}\right)$. In particular the extreme points of $J$ belong to $C_{\delta}$, so $s_{1}$ and $s_{m}$ belong to the closure of $J$. Let $\lambda_{i}$ be the geodesic that joins $s_{i}$ with $s_{i+1}$, by the construction of $C_{\delta}, \lambda_{i}$ belongs to the lamination $\Lambda$. See Figure 4 .

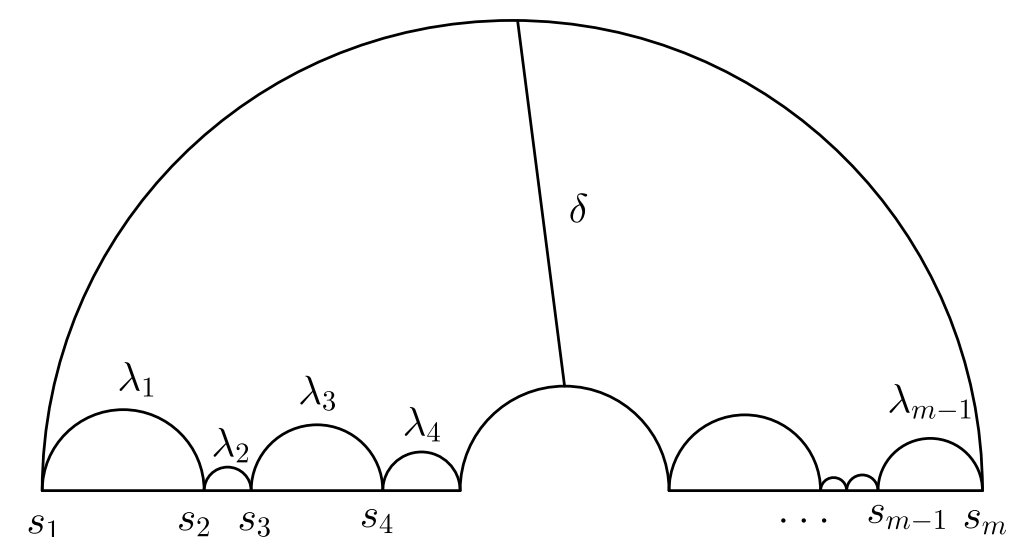

Figure 4: Figure relative to the proof of Proposition 3.5.

Due to the construction of the lamination $\Lambda$ and the limit set $C_{\delta}$, we can suppose, without loss of generality, that $J$ is the closure of a cylinder, i.e. $I_{c_{1} \ldots c_{r}}$. According to Proposition 2.1, we have $s_{1}=h_{c_{1} \ldots c_{r^{\prime}}}\left(t_{i}\right)$, for some $r^{\prime}<r$ and $i \in\{1, \ldots, k\}$.

The fact that $s_{1}$ is the end point of a cylinder implies that the points $s_{j}$, for $j \in\{2, \ldots, m\}$, are end points of cylinders. In particular $s_{1}$ and $s_{2}$ are the end points of a cylinder contained in $I_{c_{1} \ldots c_{r}}$. So $s_{1}$ and $s_{2}$ are the end points of a cylinder of the form $I_{c_{1} \ldots c_{l}}$, with $l>r$. By Proposition 2.1, $s_{1}=h_{c_{1} \ldots c_{l^{\prime}}}\left(t_{j}\right)$, for some $l^{\prime}<l$ and $j \in\{1, \ldots, k\}$, and $s_{2}=h_{c_{1} \ldots c_{l^{\prime}}}\left(t_{j}^{-}\right)$. If $r^{\prime}=l^{\prime}$ then $s_{2}=s_{m}$, so we can suppose that $r^{\prime}<l^{\prime}$. Since the maps $h_{i}$-s are the inverses of the map $f$, we have: $f^{l^{\prime}}\left(s_{1}\right)=t_{j}$, so $f^{l^{\prime}+1}\left(s_{1}\right)=f\left(t_{j}\right)=\theta$, similarly $f^{r^{\prime}+1}\left(s_{1}\right)=\theta$. Hence $f^{l^{\prime}-r^{\prime}}(\theta)=\theta$, so $\theta$ is a periodic point of $f$.

We conjecture that if $\theta$ is a $f$-periodic point then the set $C_{\delta}$ is countable.

For a given $\delta$, a transversal arc to $\Lambda$, we define $\mu(\delta)=\mathcal{M}_{s_{0}}\left(C_{\delta}\right)$ where $\mathcal{M}_{s_{0}}$ is the $s_{0}$-Hausdorff measure and $s_{0}$ is the Hausdorff dimension of $C_{\delta}$. When $f$ is a regular expanding linear map, the sets $C_{\delta}$ are the fixed points of GIFS consisting of similarities, and the GIFS satisfies the open set condition (see [8] for the definition). Due to the self-similarity of $C_{\delta}$, its Hausdorff dimension is independent of $\delta$.

The domain of $F$ can be extended to the set of equivalence classes of transverse curves to the lamination $\Lambda$. Given $\delta$ and $\delta^{\prime}$ two transverse curves to $\Lambda$, we say that $\delta \sim \delta^{\prime}$ if the end points of each curve lie in the same pair of distinct geodesics, and 
$C_{\delta}=C_{\delta^{\prime}}$. Therefore $\mu(\delta)=\mu\left(\delta^{\prime}\right)$. We extend the definition of the map $F$ to the transverse curves to $\Lambda$ and their equivalence classes. The curve $F(\delta)$ is defined as a transverse curve only to all $F(\lambda)$ where $\lambda$ are the geodesics transverse to $\delta$.

Proposition 3.6. Let $f: I \rightarrow I$ be a regular expanding linear map, such that $\theta$ not $f$-periodic; and $F: \Lambda \rightarrow \Lambda$ its induced map on the lamination $\Lambda$. Then $F$ has the property $F_{*} \mu=\left(\alpha_{1}^{s_{0}}+\cdots+\alpha_{k}^{s_{0}}\right) \mu$, where $F_{*} \mu(\delta):=\mu\left(F^{-1}(\delta)\right)$, and $\delta$ is a transverse arc to $\Lambda$.

Proof. By definition $F_{*}(\mu(\delta))=\mu\left(F^{-1}(\delta)\right)=\mathcal{M}_{s_{0}}\left(C_{F^{-1}}(\delta)\right)$. And by construction the limit set $C_{\delta}=\cap_{j \geq 0}\left(K_{j}\right)$, so $f^{-1}\left(C_{\delta}\right)=\cap_{j \geq 0} f^{-1}\left(K_{j}\right)$ and $C_{F^{-1}(\delta)}=f^{-1}\left(C_{\delta}\right)$. Since $f$ is $k$ to 1 and its inverse branches are the maps $h_{i}$, we have

$$
\begin{aligned}
& \mathcal{M}_{s_{0}}\left(C_{F^{-1}(\delta)}\right)=\mathcal{M}_{s_{0}}\left(f^{-1}\left(C_{\delta}\right)\right)=\mathcal{M}_{s_{0}}\left(\bigcup_{j=1}^{k} h_{j}\left(C_{\delta}\right)\right)=\sum_{j=1}^{k} \mathcal{M}_{s_{0}}\left(h_{j}\left(C_{\delta}\right)\right)= \\
= & \left(\alpha_{1}^{s_{0}}+\cdots+\alpha_{k}^{s_{0}}\right) \mathcal{M}_{s_{0}}\left(C_{\delta}\right)=\left(\alpha_{1}^{s_{0}}+\cdots+\alpha_{k}^{s_{0}}\right) \mu(\delta) .
\end{aligned}
$$

Therefore $F_{*} \mu=\left(a_{1}^{s_{0}}+\cdots+a_{k}^{s_{0}}\right) \mu$.

If $0<s_{0}<1$ then $\left(\alpha_{1}^{s_{0}}+\cdots+\alpha_{k}^{s_{0}}\right)>1$ since $\sum_{i=1}^{k} \alpha_{i}=1,0<\alpha_{i}<1$; so we can think $F$ as an expanding map the lamination $\Lambda$.

From these results, we have proved the following theorem:

Theorem 3.1. Let $f: I \rightarrow I$ be a regular expanding map, such that $\theta$ satisfies the hypotheses of Proposition 3.3. Then there exists a geodesic lamination $\Lambda$ on the disk with transversal measure $\mu$ and a continuous map $F: \Lambda \rightarrow \Lambda$. Moreover, if $f$ is a regular expanding linear map and $s_{0}$ is the Hausdorff dimension of a limit set on the boundary of the disk obtained by any transverse arc to $\Lambda$, satisfying $0<s_{0}<1$, then there is $\rho>1$ such that $F_{*} \mu=\rho \mu$.

\section{Examples}

Example 1: Let $\alpha_{1}=\alpha^{2}, \alpha_{2}=\alpha_{3}=\alpha \beta$ and $\alpha_{4}=\beta^{2}$, with $\alpha+\beta=1$, and $\alpha, \beta>0$; and $\theta=1-\alpha^{2} / 2$. We define $\phi_{i}(t):=\left(t-\sum_{j=0}^{i-1} \alpha_{j}\right) / \alpha_{i}$, for $1 \leq i \leq 4$, and $\alpha_{0}=0$. This example was studied in detail in [14]. The point $\theta$ is not periodic for $f$ since its itinerary is $1 \overline{3}=13333 \ldots$. The Hausdorff dimension of the limit sets $C_{\delta}$ is $s_{0}$, the solution of the equation $\alpha^{2 s}+\beta^{2 s}=1$. Moreover $0<\mathcal{M}_{s_{0}}\left(C_{\delta}\right)<\infty$. In [14], it was shown that there is an space-filling curves associated to this expanding map. The lamination of this example, with $\alpha=(-1+\sqrt{5}) / 2$ and $\beta=\alpha^{2}$, is shown in Figure 1.

Example 2: Let $\alpha_{i}=1 / 3$, for $i \in\{1,2,3\}$, and $\theta=5 / 9$. As in Example 1, $\phi_{i}(t):=\left(t-\sum_{j=0}^{i-1} \alpha_{j}\right) / \alpha_{i}$, for $i=1,2,3$, and $\alpha_{0}=0$. It can be easily checked that the itinerary of $\theta$ is $2 \overline{1}$, hence it is not periodic. The graph of this expanding map, is shown in Figure 5. Let $I_{a_{1} \ldots a_{n}}$ be a cylinder, using induction on $n$, can be checked that there are three different types of cylinder, according how are decomposed into sub-cylinders. The cylinders could have one or two connected 


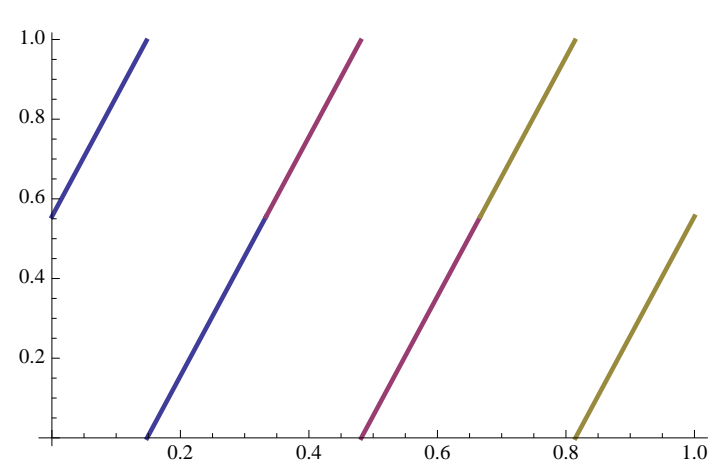

Figure 5: Graph of the expanding map of Example 2.

component. In order to understand the cylinders and how are decomposed into sub-cylinders we give the following model. Let

$$
0 \leq x_{1}<y_{1}<y_{2}<x_{2} \leq x_{3}<y_{3}<x_{4}<1,
$$

with the relations:

$$
\begin{aligned}
& \frac{\left|y_{1}-x_{1}\right|+\left|x_{4}-y_{3}\right|}{\left|x_{2}-x_{1}\right|+\left|x_{4}-x_{3}\right|}=\frac{1}{3}, \\
& \frac{\left|y_{2}-x_{2}\right|+\left|y_{3}-x_{3}\right|}{\left|x_{2}-x_{1}\right|+\left|x_{4}-x_{3}\right|}=\frac{1}{3}, \\
& \frac{\left|y_{2}-y_{1}\right|}{\left|x_{2}-x_{1}\right|+\left|x_{4}-x_{3}\right|}=\frac{1}{3} .
\end{aligned}
$$

We consider $\left[x_{1}, x_{2}\right) \cup\left[x_{3}, x_{4}\right)$ as a cylinder. There are the following possibilities:

- If $x_{2}=x_{3}$, i.e. the cylinder consists of one interval. We say that the cylinder is of type $I$.

- If $x_{2} \neq x_{3}$, i.e. the cylinder consists of two intervals. Here we allow cyclic permutations of the intervals. See Figure 6.

We say that:

- it is of type II-1 if the intervals are ordered in $[0,1)$ as in (1).

- it is of type II-2 if the order is as $0 \leq x_{3}<y_{3}<x_{4}<x_{1}<y_{1}<y_{2}<x_{2}$.

If $I_{a_{1} \ldots a_{n}}$ is of type I then its decomposition into sub-cylinders is as follows:

$$
I_{a_{1} \ldots a_{n} 1}=\left[y_{2}, y_{3}\right), \quad I_{a_{1} \ldots a_{n} 2}=\left[x_{1}, y_{1}\right) \cup\left[y_{3}, x_{4}\right), \quad I_{a_{1} \ldots a_{n} 3}=\left[y_{1}, y_{2}\right) ;
$$

where $I_{a_{1} \ldots a_{n} 3}$ and $I_{a_{1} \ldots a_{n} 1}$ are of type I, $I_{a_{1} \ldots a_{n} 2}$ is of type II-2.

If $I_{a_{1} \ldots a_{n}}$ is of type II- 1 then

$$
I_{a_{1} \ldots a_{n} 1}=\left[y_{2}, x_{2}\right) \cup\left[x_{3}, y_{3}\right), \quad I_{a_{1} \ldots a_{n} 2}=\left[x_{1}, y_{1}\right) \cup\left[y_{3}, x_{4}\right), \quad I_{a_{1} \ldots a_{n} 3}=\left[y_{1}, y_{2}\right) \text { ； }
$$

where $I_{a_{1} \ldots a_{n} 3}$ is of type I, $I_{a_{1} \ldots a_{n} 1}$ is of type II- 1 and $I_{a_{1} \ldots a_{n} 2}$ of type II-2. 


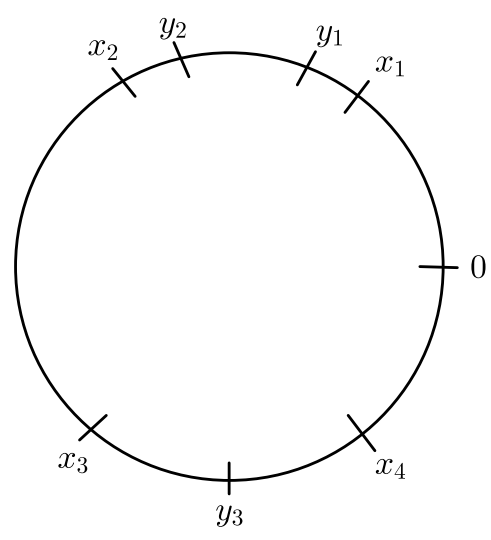

Type II-1

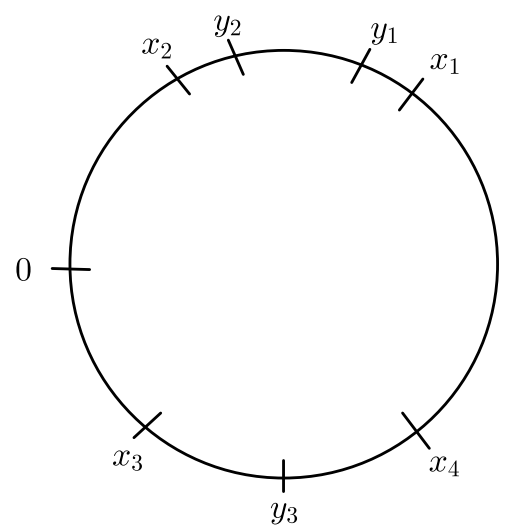

Type II-2

Figure 6: Different types of cylinders in Example 2.

If $I_{a_{1} \ldots a_{n}}$ is of type II-2 then

$$
I_{a_{1} \ldots a_{n} 1}=\left[x_{3}, y_{3}\right) \cup\left[y_{2}, x_{2}\right), \quad I_{a_{1} \ldots a_{n} 2}=\left[y_{3}, x_{4}\right) \cup\left[x_{1}, y_{1}\right), \quad I_{a_{1} \ldots a_{n} 3}=\left[y_{1}, y_{2}\right) ；
$$

where $I_{a_{1} \ldots a_{n} 3}$ is of type I, $I_{a_{1} \ldots a_{n} 1}$ is of type II- $2 I_{a_{1} \ldots a_{n} 2}$ is of type II- 1 .

We use this information to understand the construction of the limit set $C_{\delta}$. Let $\delta$ be a transverse arc whose end points are in the geodesics that joins the extreme points of a cylinder $I_{a_{1} \cdots a_{n}}$, of type II-1, see Figure 7 . The study of the case of type II-2 is done in a similar manner. So $I_{a_{1} \cdots a_{n}}=\left[x_{1}, x_{2}\right) \cup\left[x_{3}, x_{4}\right)$. Let $K_{0}$ be the closure of $I_{a_{1} \cdots a_{n}}$, i.e. $\left.\left.K_{0}=\left[x_{1}, x_{2}\right] \cup\right] x_{3}, x_{4}\right]$. In the first step of the construction of the set $C_{\delta}$, we remove from $K_{0}$ the open set $\left(y_{1}, y_{2}\right)$. So $K_{1}=$ $\left[x_{1}, y_{1}\right] \cup\left[y_{2}, x_{2}\right] \cup\left[x_{3}, x_{4}\right]$, the set $K_{1}$ consists of the closure of the cylinders $I_{a_{1} \cdots a_{n} 1}$ and $I_{a_{1} \cdots a_{n} 2}$, which are of type $I I-1$ and $I I-2$. This process defines the map $\Psi_{1,1}$ as the map that sends $I_{a_{1} \cdots a_{n}}$, of type II-1, into $I_{a_{1} \cdots a_{n} 1}$, of type II-1, using the maps $h_{1}, h_{2}$ and $h_{3}$. Similarly we get $\Psi_{1,2}$. Booth of then contract the distance by a factor $1 / 3$. We repeat this process to these new cylinders. Hence we obtained the set $C_{\delta}$ as the attractor of the graph directed iterated function systems, defined by the graph, shown in Figure 8. Since the maps $\Psi_{i, j}$ contract the distance by a factor of $1 / 3$, then the Hausdorff dimension of $C_{\delta}$ is $\log 2 / \log 3$. Furthermore it can be proved using standard techniques that $0<\mathcal{M}_{s_{0}}\left(C_{\delta}\right)<\infty$.

This analysis can be extended for any $k$ odd. In this case we consider $\alpha_{i}=1 / k$, for $1 \leq i \leq k$, and $\theta=\left(k^{2}-k-1\right) / k^{2}$. It can be checked that the itinerary of $\theta$ is $(k-1) \overline{(k-2)}$. The corresponding limit set $C_{\delta}$ has Hausdorff dimension $\log 2 / \log k$.

Example 3: Let $\alpha$ be the real root of the polynomial $x^{3}+x^{2}+x-1$. Let $\alpha_{j}=\alpha^{j}$, for $1 \leq j \leq 3$ and $\theta=\left(\alpha+\alpha^{2}\right) / 2$. As before, we define $\phi_{i}(t):=\left(t-\sum_{j=0}^{i-1} \alpha_{j}\right) / \alpha_{i}$, for $i=1,2,3$, and $\alpha_{0}=0$. The itinerary of $\theta$ is $1 \overline{12}$, which satisfies the hypothesis (b) of Proposition 3.3. Some dynamical properties of this map were studied in [3]. The geodesic lamination was introduced and studied in detail in [12]. The lamination is shown in Figure 10. In this example the dimension of the limit sets is calculated in detail and the value for $s_{0}$ is $\log \rho / \log \alpha$, where $\rho$ is the real 




Figure 7: The construction of the set $C_{\delta}$ in Example 2.

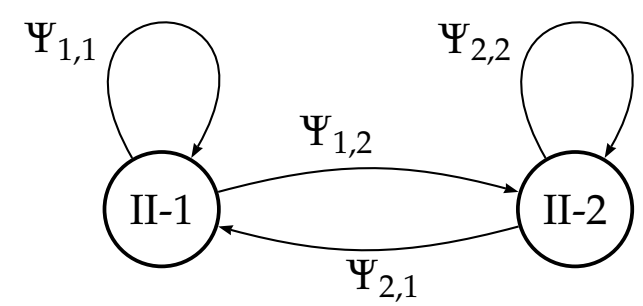

Figure 8: The graph-directed IFS that defines the limit set $C_{\delta}$ of Example 2.

root smaller than one, in absolute value, of the polynomial $x^{4}+2 x^{3}-1, s_{0}$ is approximately 0.5466 . This construction is related to the Rauzy fractal, for details see $[1,12,2]$. This construction can be generalized to $k$ maps, i.e. $\left\{\phi_{1}, \ldots, \phi_{k}\right\}$. Where $\alpha_{j}=\alpha^{j}$, for $1 \leq j \leq k$, and $\alpha$ is the real root smaller than one, in absolute value of the polynomial $x^{k}+x^{k-1}+\cdots+x-1$, for details see [12].

Example 4: Here we will consider a non-linear version of Example 3. Let $\phi_{i}: I_{i} \rightarrow$ $I$ given by

$$
\phi_{i}(t)=\frac{\exp \left(t-\sum_{j=0}^{i-1} \alpha_{j}\right)-1}{\exp \left(\alpha_{i}\right)-1}
$$

where $\alpha_{i}=\alpha^{i}$, for $1 \leq i \leq 3$ and $\alpha_{0}=0$, as in Example 3. Numerical evidence, suggests that the point $\theta$ is not periodic, and part of its itinerary is $1122231121 \ldots$.. The graph of the expanding map is shown in Figure 11 and the corresponding geodesic lamination is shown in Figure 10.

Example 5: Here we will consider another linear example, but $\theta$ periodic. Let $\alpha_{i}=1 / 3$, for $i \in\{1,2,3\}$, and $\theta=1 / 6$, whose itinerary is $\overline{123}$. It can be easily checked in this example that $\theta$ is not a degenerate geodesic. The corresponding lamination is shown in Figure 12. 


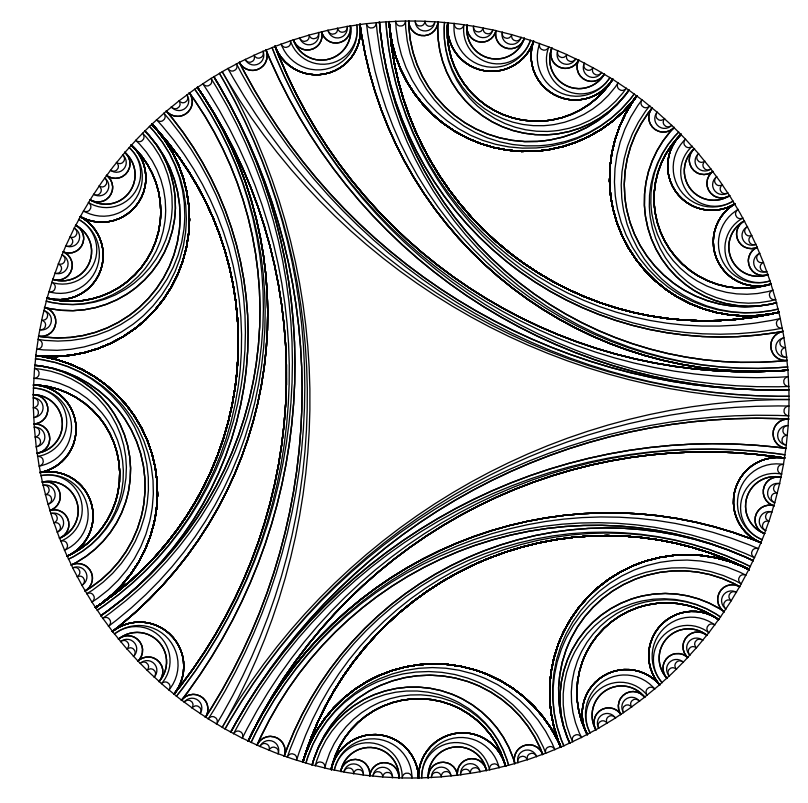

Figure 9: Geodesic lamination of Example 2.

\section{Open problems and some questions}

1. Is it true that if $\theta$ is a periodic point for the map $f$, then the limit set $C_{\delta}$ is countable, for all transversal arcs $\delta$ ?

2. Given a transversal arc $\delta$; for which values of $\theta, C_{\delta}$ is a Cantor set with $0<\mathcal{M}_{s}\left(C_{\delta}\right)<\infty$ ?

3. Let $\alpha_{j}, 1 \leq j \leq k$, as considered previously, i.e. $0<\alpha_{j}<1$ and $\sum_{j=1}^{k} \alpha_{j}=1$, and $\theta \in(0,1)$. Let $f_{\theta}$ be the linear expanding map associated to $R_{\theta}$ and the linear maps $\phi_{j}: I_{j} \rightarrow I, \phi_{j}(t):=\left(t-\sum_{i=1}^{j-1}\right) / \alpha_{j}$. Let $s_{\theta}$ be the Hausdorff dimension of the limit sets of the transversal to the lamination $\Lambda$. We would like to know the behaviour of the function $\theta \mapsto s_{\theta}$.

4. Does exist a regular expanding (linear) map with $\theta$ non-periodic and not a degenerate geodesic?

5. Characterization of the degenerate geodesics.

6. Give necessary and sufficient conditions on the map $f$, so that the elements of $\mathcal{I}$ have a bounded number of connected components. 

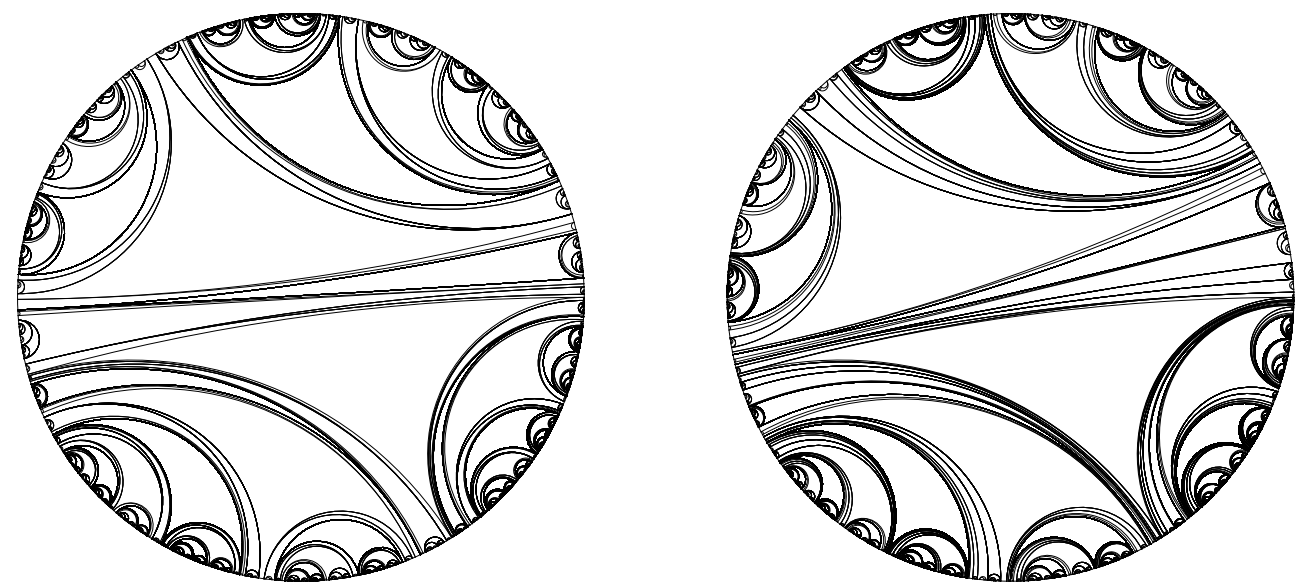

Figure 10: Geodesic lamination of Example 3 (left) and Example 4 (right).

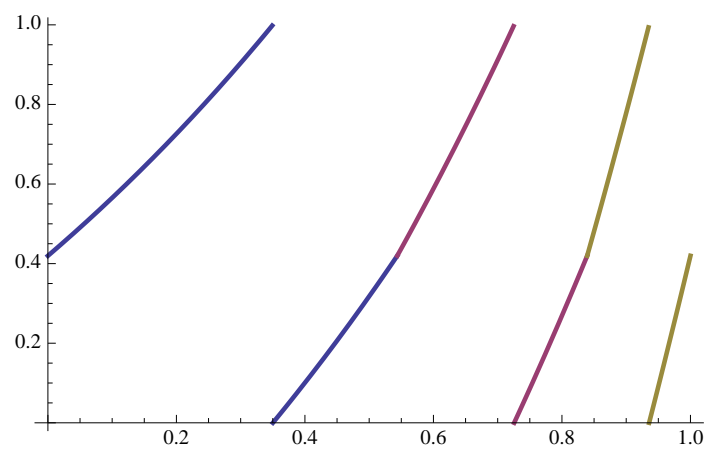

Figure 11: Graph of the expanding map of Example 4.

\section{References}

[1] P. Arnoux, Un exemple de semi-conjugaison entre un échange d'intervalles et une rotation sur le tore, Bull. Soc. Math. France, 116 (1988), 489-500.

[2] P. Arnoux, J. Bernat and X. Bressaud, Geometric Models for substitutions. Experimental Mathematics, 20 (2011), 97-127.

[3] P. Arnoux and J.C. Yoccoz, Construction de difféomorphismes pseudoAnosov, C.R.A.S. 292 (1981), 75-78.

[4] C. Bandt and K. Keller, Symbolic dynamics for the angle-doubling on the circle: I. The topology of locally connected Julia sets, Ergodic Theory and Related Topics (Lecture Notes in Mathematics 1514), U. Krengel at al. eds., Springer, Berlin, 1-23.

[5] F. Bonahon, Geodesic laminations on surfaces, in: Laminations and foliations in dynamics, geometry and topology (Stony Brook, NY, 1998), 1-37, Contemp. Math. 269, Amer. Math. Soc., Providence, RI, 2001. 




Figure 12: Geodesic lamination of Example 5, where $\theta$ is periodic.

[6] A. J. Casson and S. A. Bleiler, Automorphisms of Surfaces after Nielsen and Thurston, Cambridge University Press, 1988, Cambridge.

[7] K. Falconer, Fractal Geometry. Mathematical Foundations and Applications, John Willey \& Sons, Chichester, 1990.

[8] K. Falconer, Techniques in Fractal Geometry, John Willey \& Sons, Chichester, 1997.

[9] K. Keller, Symbolic dynamics for the angle-doubling on the circle, III: Sturmian sequences and the quadratic map, Ergod. Th. and Dynam. Sys., 14 (1994), 787-805.

[10] K. Keller, Invariant factors, Julia equivalences and the (abstract) Mandelbrot set, Lecture Notes in Mathematics, 1732, Springer, Berlin, 2000.

[11] W. de Melo and S. van Strien, One-Dimensional Dynamics, Springer, New York, 1993.

[12] V.F. Sirvent, Geodesic laminations as geometric realizations of Pisot substitutions, Ergod. Th. and Dynam. Sys., 20 (2000), 1253-1266.

[13] V.F. Sirvent, Geodesic laminations as geometric realizations of ArnouxRauzy sequences, Bull. Belg. Math. Soc. Simon Stevin, 10 (2003), 221-229.

[14] V.F. Sirvent, Space-filling curves and geodesic laminations, Geom. Dedicata, 135 (2008), 1-14.

[15] V.F. Sirvent, Space-filling curves and geodesic laminations. II: Symmetries, Monatsh. Math., 166 (2012), 543-558. 
[16] W.P. Thurston, On the geometry and dynamics of iterated rational maps, in Complex Dynamics, Families and Friends, D. Schleicher ed., A.K. Peters Ltd, Wellesley, 2009, pp. 1-110.

Departamento de Matemáticas,

Universidad Simón Bolívar, Apartado 89000, Caracas 1086-A,

Venezuela.

email: vsirvent@usb.ve

url: http://www.ma.usb.ve/〜vsirvent 